\title{
Beurteilung bestehender Stahltragwerke: Empfehlungen zur Abschätzung der Restnutzungsdauer
}

Die zunehmende Bedeutung der Nachhaltigkeit im Bauwesen wird in Zukunft das Bauen im Bestand gegenüber Neubauten in den Vordergrund rücken. Dies gilt auch für den Brückenbau, wo das Qualitätsmanagement seit langem Brücken von ihrem Entstehungsgang über den Betrieb bis in die Entsorgung begleitet. Die starke Vergrößerung der Frachtvolumina für Straße und Schiene und die zunehmende Überalterung des Brückenbestandes fragen danach, ob die bestehenden Brücken für die veränderten Betriebsbedingungen noch geeignet sind und wie sie und ob sie dafür instandgesetzt werden könnten.

Für die erforderlichen Instandhaltungs- und Instandsetzungsmaßnahmen oder einen eventuellen Ersatz durch einen Neubau ist aber die Frage der Restnutzungsdauer von entscheidender Bedeutung. Es ist daher nicht von ungefähr, dass sich Baulastträger auf nationaler und internationaler Ebene frühzeitig zusammengetan haben, um nach geeigneten Methoden der Bestimmung der Sicherheit und Restnutzungsdauer bestehender Brücken zu forschen. Auch die internationalen technisch-wissenschaftlichen Verbände wie EKS und fib sowie die Standard-Organisationen ISO und CEN sind der Frage eines internationalen Austauschs und der Vereinheitlichung der Methoden nachgegangen. Für Stahlbrücken hat insbesondere das technische Komitee 6 Ermüdung - der EKS Anregungen aufgegriffen, aus den verschiedenen nationalen Erfahrungen und dem vorhandenen Wissen technische Empfehlungen für die Beurteilung bestehender Stahlbrücken zu entwickeln [1], die sich als Grundlage für die von CEN/TC 250 - das CEN-Komitee für die Bearbeitung der Eurocode - geplante Erweiterung der Eurocode zur Instandhaltung und Instandsetzung des Brückenbestandes eignen.

Das Ergebnis ist ein technisch-wissenschaftlicher Bericht, der im Rahmen der Zusammenarbeit der europäischen Kommission (DG-Research - Joint Research Centre JRC) mit der EKS als Basispapier für eine zukünftige Eurocode-Erweiterung veröffentlicht wurde und schon jetzt als Empfehlung für eine Vorgehensweise nach dem Stand der Technik verwendet werden kann. Der vorliegende Beitrag behandelt die Grundzüge dieses Berichtes [1], der vom e-bookshop der Kommission kostenfrei herunter geladen werden kann.

\footnotetext{
Assessment of existing steel structures: recommendations for estimation of remaining fatigue life. The growing importance of sustainability affects new markets for the construction industry; the rehabilitation and refurbishment of existing buildings and civil engineering works is getting more and more into the focus of construction activities. This also applies to bridges as well where the quality management comprising the full life of a bridge has a long tradition. For bridges in particular the enormous increase of freight-volume on roads and rails and the growing age of the
}

bridges cause the question, whether the existing structures are still fit for use and whether or not they can be retrofitted and if so how that could be done.

For decision making concerning measures for maintenance and refurbishment of a bridge or its substitution by a new bridge the value of the residual service life is very important.

Therefore the bridge authorities have been cooperating for a long time to develop appropriate methods for determining the residual safety and service life of existing bridges. Also the International Technical-Scientific Organisations as the ECCS and fip as well as Standard Organisations as ISO and CEN have cared for an international exchange of views and the harmonisation of methods. For steel bridges in particular the Technical Committee 6 - Fatigue - of the ECCS has taken over the task to develop a technical guidance for the assessment of existing steel bridges on the grounds of national experiences and the present state of the art [1]. This guidance has been prepared to serve as a basis for CEN/TC 250 - the CEN-Technical Committee responsible for the structural Eurocodes - for future further development of these Eurocodes to include the assessment and retrofitting of existing structures. The result of the development of ECCS-TC 6 is a technical-scientific report which has been published by the Commission of the EU (DG-Research - Joint Research Centre - JRC) in the frame of a cooperation agreement between the JRC and the ECCS. This report may be used as a technical recommendation as long as the revision of the Eurocodes has not been carried out. This contribution gives the main information from the JRC-ECCSreport [1], that can be downloaded free of charge from the e-bookshop of the Commission.

\section{Einleitung}

Mit dem Ziel, die Kenntnisse und die Erfahrungen auf dem Gebiet der Beurteilung bestehender Stahltragwerke in Empfehlungen zusammenzufassen, wurde 1993 im Technischen Komitee 6 „Ermüdung“ der Europäischen Konvention für Stahlbau (EKS) eine Arbeitsgruppe „Restlebensdauer" eingerichtet. Bis zum Jahr 2000 wurden die Arbeiten der Arbeitsgruppe von Dr. Klaus Brandes, ehemals an der Bundesanstalt für Materialforschung und -prüfung (BAM) in Berlin, koordiniert. Danach wurde der Vorsitz der Arbeitsgruppe von Dr. Bertram Kühn, ehemals am Lehrstuhl für Stahlbau an der RWTH Aachen unter der Leitung von Prof. Sedlacek tätig, übernommen, der die Arbeitsgruppe bis zur Veröffentlichung der Empfehlungen [1] Anfang 2008 geleitet hat. 
Während der vergangenen Jahrzehnte wurden die Instrumente zur Inspektion und Zustandsbewertung des vorhandenen Brückenbauwerksbestands zunehmend weiterentwickelt und gut organisiert. In vielen Ländern sind mittlerweile Brücken-Management-Systeme (BMS) im Einsatz. Dennoch sind Empfehlungen zur Beurteilung der Ermüdungssicherheit von alten Eisen- und Stahlbrücken von großem Interesse für viele Baulastträger. Die hier vorgestellten Empfehlungen geben hilfreiche Hinweise zur Analyse der Ermüdungssicherheit, zur Identifizierung und Charakterisierung von alten metallischen Werkstoffen, zur Anwendung von Verstärkungsmaßnahmen für bestehende Stahlkonstruktionen und zur Instandsetzung bereits initiierter Schädigungen.

Mit der rasanten Entwicklung neuer Materialien, Berechnungsmethoden und Bauweisen in der zweiten Hälfte des 19. Jahrhunderts und dem Übergang zum 20. Jahrhundert wurden viele Eisen- und Stahlbrücken gebaut, die z. T. noch bis heute benutzt werden. Gerade die alten metallischen Werkstoffe und ihre Eigenarten sind heute vielen an der Unterhaltung, der Instandsetzung und der Überwachung von bestehenden Brücken Beteiligten nicht mehr geläufig, und häufig sind auch die Methoden zur Abschätzung der verbleibenden Restlebensdauer unbekannt.

Es ist einerseits eine Tatsache, dass die Beurteilung der Ermüdungssicherheit im 19. Jahrhundert nicht Bestandteil der Bemessung der alten Brücken war. Hinzu kommt insbesondere für Straßenbrücken, dass die Verkehrslastgeschichte für nachträgliche Ermüdungsnachweise nicht oder nur sehr unzureichend dokumentiert ist. Auf der anderen Seite wurden Änderungen an den Tragwerken, Verstärkungen oder Reparaturmaßnahmen nicht immer in den Bestandsunterlagen dokumentiert oder gingen in den letzten 100 Jahren, z. B. während der beiden Weltkriege, verloren.

Vor diesem Hintergrund und der Tatsache, dass die Verkehrsbeanspruchungen der Tragwerke z. T. drastisch zugenommen haben oder diese um zusätzliche Fahrspuren ergänzt wurden, ist offenkundig, warum die Untersuchung der Ermüdungssicherheit so wesentlich geworden ist.

Für die Bewertung von Tragwerken sind die vorhandene Ermüdungsfestigkeit und die Analyse der bisherigen wie auch zukünftigen Verkehrsbelastung sehr wichtig, um z. B. in Form eines akkumulierten Schadensnachweises für die Vergangenheit mit Hilfe inzwischen gut erprobter Berechnungsmethoden die verbleibende Restlebensdauer ermitteln zu können.

Werden bei Inspektionen bereits initiierte Ermüdungsrisse gefunden oder ist die Lastgeschichte gänzlich unbekannt, können aufwendigere Berechnungsmethoden wie die der linear-elastischen Bruchmechanik angewandt werden. Der Vorteil dieser Methode ist der, dass die bruchmechanische Berechnung von einem angenommenen (fiktiven) oder bei Inspektionen tatsächlich detektierten Anriss $\mathrm{a}_{0}$ ausgeht und damit die stark streuende Rissinitiierungsphase vernachlässigt. Die Annahme fiktiver Anrisse dient als Ausgangspunkt für die Berechnung des stabilen Risswachstums. Die gesamte Vorgeschichte eines Tragwerkes bis zum Erreichen der fiktiven postulierten Anrisslänge $\mathrm{a}_{0}$ ist somit nicht relevant. Die Dauer des stabilen Risswachstums vom Anfangsriss bis zum Erreichen einer kritischen Risslänge $a_{c}$ (teilweise auch $a_{d}$ genannt) kann unter bestimmten Voraussetzungen als sicheres Betriebszeitintervall verstanden werden, das sich zumindest theoretisch unendlich lange bis zu dem Zeitpunkt wiederholen lässt, zu dem bei einer Inspektion am Ende des sicheren Betriebszeitintervalls ein Ermüdungsanriss gefunden wird. Liegt ein solcher Anriss vor, liefert die bruchmechanische Berechnung den Zeitraum, innerhalb dessen der Schaden spätestens zu reparieren ist.

Tatsächlich bleibt bei fiktiver Anrissannahme ein großer Anteil der gesamten Lebensdauer eines Tragwerks, die Rissinitiierungsphase, unberücksichtigt. Gleichzeitig besteht aber die Möglichkeit, selbst noch nach Ablauf der theoretischen Lebensdauer eines Tragwerkes eine sichere Weiternutzung über große Zeiträume nachzuweisen.

\section{Beurteilungsschema \\ 2.1 Anwendungsgrenzen}

Die Beurteilung der Ermüdungssicherheit eines bestehenden Tragwerks führt gemäß den Empfehlungen der EKS [1] zu einer Feststellung der Sicherheit eines Tragwerks unter wechselnder Beanspruchung für die ermittelte verbleibende Restnutzungsdauer. Die Anwendbarkeit der hier aufgeführten Empfehlungen ist auf die Nutzung von Tragwerken unter normalen Umweltbedingungen, d. h. dem Fehlen übermäßig korrosiver Umgebungsbedingungen und auf eine Temperaturskala zwischen $-40^{\circ} \mathrm{C}$ und $150^{\circ} \mathrm{C}$, begrenzt. Eine Anwendung im Kurzzeitfestigkeitsbereich, d. h. bei Beanspruchungen mit wenigen Lastwechseln bei gleichzeitig hohen Beanspruchungsgrößen, wie sie z. B. bei seismischen Aktivitäten auftreten, ist ebenfalls nicht möglich. Das zu beurteilende Tragelement muss zudem für Inspektionen zugänglich und somit auf seinen aktuellen Zustand hin überprüfbar sein.

\subsection{Vorgehensweise}

Für die Beurteilung der Ermüdungssicherheit bestehender Tragwerke wird eine phasenweise Vorgehensweise vorgeschlagen, s. Bild 1. Diese beruht in ihren Grundzügen auf einem vom Joint Committee for Structural Safety (JCSS) entwickelten Verfahren [2], das sein Hauptaugenmerk auf bestehende Stahlbrücken legt, die wechselnden Beanspruchungen infolge des Verkehrs ausgesetzt sind.

In Fällen, in denen die Beurteilung in einer der nachfolgend genannten Phasen eine ausreichende Restlebensdauer ausweist, können die darauf folgenden Phasen ausgesetzt werden. Im Einzelnen werden folgende vier Phasen vorgeschlagen:

\section{Phase I. Voruntersuchung:}

Ausräumung von vorhandenen Zweifeln über die Sicherheit des zu untersuchenden Tragwerks unter Verwendung deterministischer Methoden entsprechend dem Stand der Technik. Dabei werden Informationen aus vorhandenen Bauwerksprüfungen, aus eigenen Sichtprüfungen und, falls vorhanden, aus Brücken-Management-Systemen verwendet. Die Untersuchungen haben den Charakter einer groben Abschätzung, mit dem Ziel, abzuwägen, ob weitere detailliertere Untersuchungen erforderlich sind. In dieser Phase wird festgelegt, auf welche maßgebenden Bereiche die Untersuchungen beschränkt werden können. Der Bau- 
lastträger wird vom erfahrenen Ingenieur oder Brückenprüfingenieur über die Ergebnisse der Voruntersuchungen in einem ersten Bericht unterrichtet.

\section{Phase II. Detaillierte Untersuchung:}

Diese Untersuchungen werden von einem erfahrenen Ingenieur ggf. unter Hinzuziehung spezialisierter Institute und Fachleute durchgeführt. Es werden aktuelle Informationen am Tragwerk und realistische Verkehrsbeanspruchungen ermittelt und in die Berechnungen unter Nutzung verbesserter semi-probabilistischer Berechnungsmodelle aufgenommen. Der Sicherheitsindex aus der Differenz zwischen Belastung und Belastbarkeit beschreibt die Zuverlässigkeit. Die Ergebnisse dieser aktualisierten Untersuchungen sind dem Baulastträger in einem zweiten Bericht mitzuteilen. Falls die Ergebnisse dieser Phase zu keiner ausreichenden Ermüdungssicherheit des untersuchten Tragwerks führen, können dem Baulastträger verschiedene Vorschläge als Entscheidungshilfe zum weiteren Vorgehen gegeben werden.

Phase III. Fachkundige Untersuchung:

In dieser Phase wird das probabilistische Konzept angewandt. Alle Variablen gehen mit ihrer tatsächlichen statistischen Verteilung in die Berechnung ein. Neben statistischen Größen für Materialeigenschaften und Lasten wird ein verfeinertes, $\mathrm{z}$. B. in realen Messungen ermitteltes statisches Modell verwendet. Es sind ausschließlich fachkundige Spezialisten im Umgang mit bestehenden Tragstrukturen anzufragen. Die Restnutzungsdauer kann sowohl mittels probabilistischer als auch bruchmechanischer Untersuchungsmethoden ermittelt werden. Zur Detektierung möglicher Anrisse können an hoch beanspruchten Querschnitten oder an Verbindungen, die sich in anderen Bauwerksabschnitten als besonders ermüdungsanfällig erwiesen haben, zielgerichtet zerstörungsfreie Prüfmethoden (ZfP) eingesetzt werden. Auch diese Phase wird mit einem Bericht an den Baulastträger abgeschlossen.

Phase IV. Weiterführende Maßnahmen:

In Phase IV trifft der Baulastträger die Entscheidung für die weitere Nutzung. Das betrachtete Tragwerk wird unter Berücksichtigung der Ergebnisse aus den Phasen I bis III für den geplanten weiteren Verwendungszweck und die vorgesehene weitere Nutzungsdauer ertïchtigt, repariert und/oder verstärkt, die Lasten werden reduziert, es wird verstärkt inspiziert, oder das Bauwerk wird durch einen Neubau ersetzt. Eine weitere Möglichkeit ist eine höhere Inspektionsfrequenz der ermüdungsgefährdeten Details. Ein Abschlussbericht fasst die Ergebnisse aller Arbeitsschritte zusammen, listet die technisch möglichen Maßnahmen auf und gewichtet diese im Hinblick auf Ausführbarkeit und technischen Aufwand. Dieser Bericht enthält damit alle Informationen, die der Baulastträger für eine wirtschaftliche Entscheidung über die zu ergreifenden Maßnahmen braucht.

Häufig führt die Anwendung der vorgeschlagenen Vorgehensweise bei der Beurteilung bestehender Tragwerke zu einer über die theoretische Lebensdauer hinausgehenden Nutzungsdauer und damit zu einem Aufschub von Bauin- vestitionen. Der Baulastträger gewinnt die damit verbundenen Zinseffekte, oder er hat die Möglichkeit, die Mittel anderweitig einzusetzen. Das Beurteilungsschema klärt also $\mathrm{ab}$, ob das Tragwerk ohne weitere Maßnahmen für eine bestimmte Nutzung und eine bestimmte Restlebensdauer ausreichend sicher ist oder, wenn nicht, welche Maßnahmen für eine sichere Weiternutzung zu ergreifen sind oder ob das Tragwerk ersetzt werden muss. Damit hilft das in [1] empfohlene Schema, die beste Strategie für die optimalen Lebenszykluskosten zu finden, s. Bild 1.

Die Empfehlungen in [1] geben detaillierte Informationen zu jeder Phase des Beurteilungsschemas. Hierbei werden in Phase I konservative Annahmen sowohl für die Berechnung der Beanspruchung als auch für die Beurteilung der Beanspruchbarkeit verwendet. So ist z. B. die Kerbfallklasse, die für die Bewertung von genieteten Tragwerken empfohlen wird, ohne weitere Unterscheidungen hinsichtlich des zu beurteilenden Tragglieds und dessen konstruktiver Form einheitlich als konservative Abschätzung angegeben. Für die Belastungsgeschichte sollen ebenfalls nur vereinfachte konservative Abschätzungen benutzt werden, in der Form, dass eine Beanspruchung mit den heutzutage in einer Neubemessung anzusetzenden Verkehrslasten für die gesamte Vergangenheit der Brücke unterstellt wird. Der Zustand des Tragwerks, z. B. vorhandene Abrostungen, Schäden aus Anprall oder nicht ausgebesserte Kriegsschäden sind durch einfache Abschätzungen zu berücksichtigen. Wie in Bild 1 dargestellt, wird die Ermüdungssicherheitsrate $\mu_{\text {fat }}$ für den Grenzzustand, hier das Erreichen des noch sicheren Lebensdauerendes, eingeführt. Die kritischsten Details haben die niedrigsten Sicherheitsraten. Die Ermüdungssicherheitsrate wird wie folgend ausgedrückt:

$$
\mu_{\text {fat }}=\frac{\gamma_{\mathrm{Ff}} \cdot \Delta \sigma_{\mathrm{E}, 2}}{\Delta \sigma_{\mathrm{c}} / \gamma_{\mathrm{Mf}}}
$$

$\mu_{\text {fat }} \quad$ Ermüdungssicherheitsrate

$\gamma_{\mathrm{Ff}} \quad$ Teilsicherheitsfaktor für die äquivalente konstante Spannungsschwingbreite

$\Delta \sigma_{\mathrm{E}, 2}$ äquivalente konstante Spannungsschwingbreite bezogen auf 2 Mio. Zyklen

$\Delta \sigma_{\mathrm{c}} \quad$ Referenzwert der Ermüdungsfestigkeit bei 2 Mio. Zyklen (Detailkategorie)

$\gamma_{\mathrm{Mf}} \quad$ Teilsicherheitsfaktor für die Ermüdungsfestigkeit $\Delta \sigma_{\mathrm{c}}$

Auf die Ermüdungsfestigkeit $\Delta \sigma_{\mathrm{c}}$ für genietete Details wird später noch eingegangen.

In Phase II wird eine detaillierte Untersuchung durchgeführt, um die Informationen aus Phase I zu aktualisieren. In dieser Untersuchung werden verfeinerte Eingangsdaten für das Strukturmodell, realistischere Lasten und dynamische Faktoren verwendet. Vorhandene Querschnittsschwächungen sind im Detail aufzunehmen und im Berechnungsmodell zu berücksichtigen. An ermüdungskritischen Details wird die verbleibende Restlebensdauer mit Hilfe der Schädigungsakkumulation nach Palmgren-Miner abgeschätzt [4]:

$\sum \frac{\mathrm{n}_{\mathrm{i}}}{\mathrm{N}_{\mathrm{i}}}=1$ 


\section{Anlass für eine Beurteilung: Nutzungsänderung, Lebensdauer rechnerisch erreicht, Schäden entdeckt}

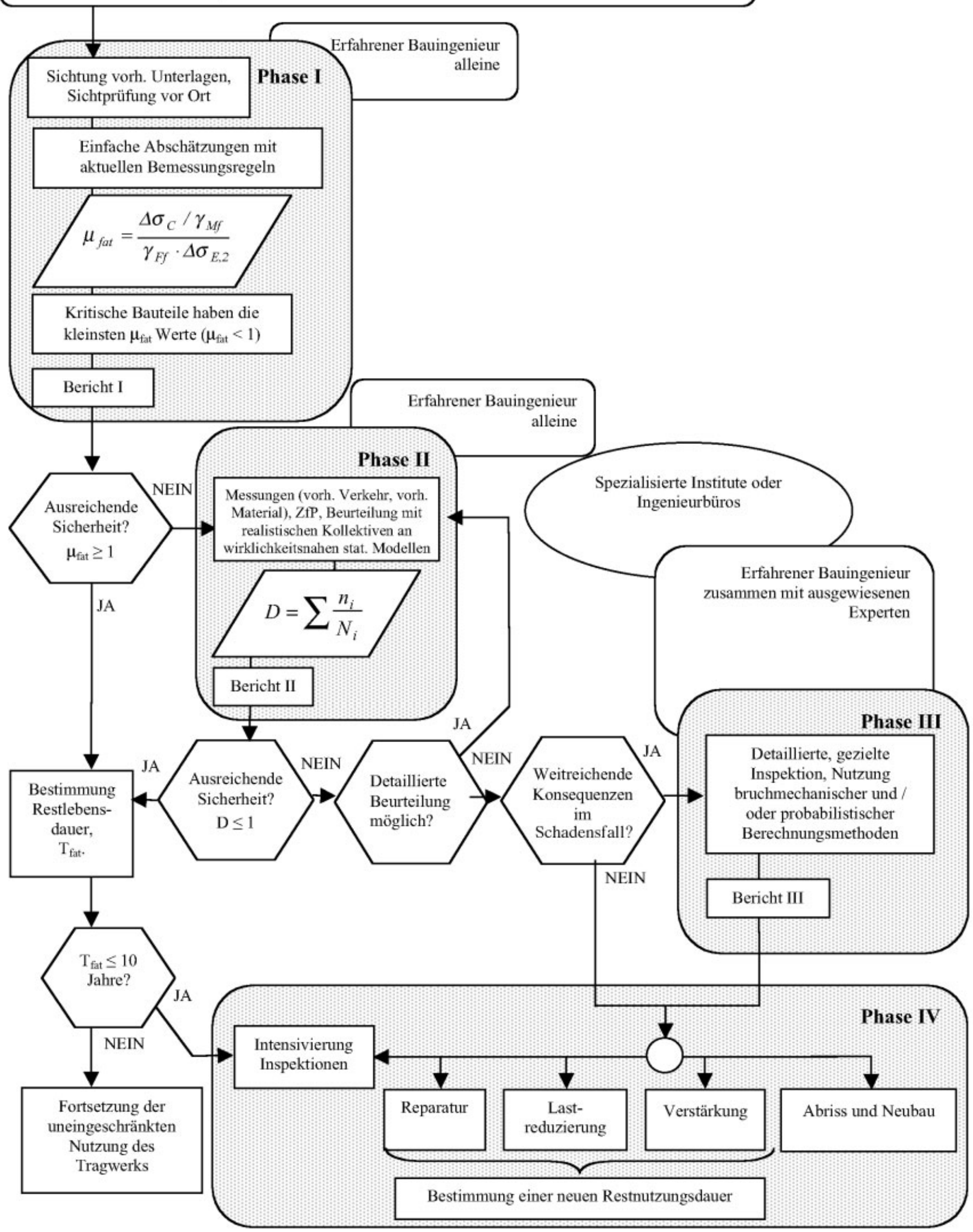

Bild 1. Schrittweises Beurteilungsschema für ermüdungsbeanspruchte Tragwerke

Fig. 1. Stepwise procedure for fatigue assessment 
$\mathrm{n}_{\mathrm{i}} \quad$ tatsächliche Zyklenanzahl bei der Spannungsamplitude $\mathrm{i}$

$\mathrm{N}_{\mathrm{i}}$ theoretische Ermüdungslebensdauer bei der Spannungsamplitude $\mathrm{i}$

Nähere Informationen zur Anwendung der Schädigungsakkumulation z. B. nach Palmgren-Miner kann [16] entnommen werden. Wenn die Auswertung nach dem Wöhlerkurvenkonzept (mit Ermüdungsfestigkeitskurven) keine ausreichende Restlebensdauer mehr ergibt, ist eine weiterführende Untersuchung nach Phase III durchzuführen. Diese umfasst Messungen am Bauwerk, die Ermittlung statistisch abgesicherter Materialkennwerte und auch die Untersuchung ermüdungskritischer Details auf eventuelle Anrisse mit Hilfe zerstörungsfreier Prüfmethoden. Das sind die Eingangswerte für eine Berechnung mit Hilfe der linear-elastischen Bruchmechanik (linear-elastic fracture mechanical approach, LFMA). Die Berechnung mit Hilfe der Bruchmechanik kommt auch zur Anwendung, wenn entweder ein tatsächlicher Riss entdeckt wurde oder wenn ein erkennbarer Anriss theoretisch postuliert wird. Die stabile Rissausbreitung wird nach dem Gesetz von Paris beschrieben. Der zyklische Spannungsintensitätsfaktor $\Delta \mathrm{K}$ für die Einwirkung, s. Gl. (3), ist eine materialunabhängige Größe.

$$
\Delta \mathrm{K}=\mathrm{Y} \cdot \Delta \sigma \sqrt{\pi \cdot \mathrm{a}}
$$

$\Delta \sigma \quad$ angewandte zyklische Spannungsbreite

Y Geometriefaktor (i. d. R. aus der Literatur, zum Beispiel von Murakami [15])

$\Delta \mathrm{K}$ zyklischer Spannungsintensitätsfaktor

a Risslänge

Die materialspezifischen Konstanten $\mathrm{m}$ und $\mathrm{C}$ sowie der ebenfalls materialspezifische Widerstandswert (Schwellenwert) des Spannungsintensitätsfaktors $\Delta \mathrm{K}_{\text {th }}$, können in standarisierten Risswachstumsversuchen ermittelt werden. Das sichere Betriebszeitintervall wird aus dem stabilen Risswachstum bis zum Erreichen einer kritischen Risslänge $a_{c}$ zurückgerechnet auf eine ertragbare Zyklenzahl, die das sichere Inspektionsintervall charakterisiert.

Die Empfehlung bietet die Basis für den Betriebszeitintervallnachweis. Zum Ende jedes Intervalls wird eine Inspektion durchgeführt, und die kritischen Details werden in Bezug auf Rissentstehung kontrolliert. Falls keine Risse gefunden werden, wird die Brücke für ein nächstes Betriebzeitintervall bis zur nächsten Inspektion als sicher eingestuft. Im Fall, dass während der Inspektion tatsächlich kritische Ermüdungsrisse festgestellt wurden, sind Abhilfemaßnahmen einzuleiten, z. B. der Austausch des gerissenen Elements, aber auch Bohrungen zum Stoppen des Rissfortschrittes. Für den Fall, dass für den Austausch oder die notwendigen Instandsetzungsmaßnahmen keine Mittel zur Verfügung stehen, kann auch ein neues, kürzeres Inspektionsintervall errechnet werden. Die tatsächliche Risslänge wird gemessen, und mit dieser Information kann man eine kritische Risslänge und ein neues Inspektionsintervall festlegen [5]. Die Ergebnisse der Phase III werden dem Brückeneigentümer oder Baulastträger in einem Bericht für seine weitere Entscheidungsfindung vorgelegt.
In Phase IV werden alle verbleibenden Möglichkeiten ausgeschöpft, um zu einer Entscheidung über den weiteren Betrieb, eine Reparatur und Verstärkung, eine Nutzungsänderung oder einen Ersatz zu kommen. Die Maßnahmen in Phase IV werden auch durch gesellschaftspolitische und soziale Kriterien sowie durch Ökonomie und durch mögliche Schadensfolgen in Verbindung mit der Relevanz des Bauwerks innerhalb eines Transportnetzwerkes bestimmt. Im abschließenden Bericht werden Angaben zum ermüdungsrelevanten Belastbarkeitsfaktor, z. B. für Eisenbahnbrücken $\beta_{D, U I C}$ oder zum Sicherheitsindex $\beta$ und zur Versagenswahrscheinlichkeit gemacht. Für weitere Details siehe [1]. Die Ergebnisse der Phase IV werden für den Brückeneigentümer in einem Schlussbericht zusammengestellt.

\section{Beanspruchbarkeit 3.1 Allgemeines}

Um Informationen über den Widerstand eines Tragwerkes zu erhalten, können sowohl Messungen am Tragwerk als auch Materialuntersuchungen durchgeführt werden. Damit zeigt sich ein großer Vorteil gegenüber der Bemessung von Neubauten, bei denen das zu erwartende Tragverhalten und die $\mathrm{zu}$ erwartenden Materialkenngrößen vorab geschätzt und angenommen werden müssen. Ferner ist bekannt, dass Materialkenngrößen von alten Stählen, die seinerzeit verwendeten Bemessungsregeln und die Verbindungstechniken von den heutzutage üblichen und bekannten deutlich abweichen können. Dies ist der Grund, warum die Bemessung, das Material und die konstruktive Durchbildung zunächst zu studieren sind, um gegenüber den bekannten Größen für die Erstellung von Neubauten zusätzliche notwendige Erkenntnisse zu erhalten.

Wie beim Neubau werden bei einer Berechnung bestehender Brücken die Beanspruchungen (Lasten) der Beanspruchbarkeit (Material) gegenübergestellt. Sowohl bei den zu erwartenden äußeren Beanspruchungen als auch bei den Materialkennwerten werden statistische Streuungen durch Teilsicherheitsfaktoren abgesichert.

\subsection{Identifikation des Materials}

Es können verschiedene Quellen genutzt werden, um Informationen über den Materialwiderstand $\mathrm{zu}$ erhalten, z. B. alte Materialtabellen oder standardisierte Versuche mit altem Originalmaterial zur Bestimmung:

- der unteren und oberen Streckgrenze $R_{e L}, R_{e H}$ und der Zugfestigkeit $\mathrm{R}_{\mathrm{m}}$

- des Elastizitätsmoduls E

- der chemischen Zusammensetzung, insbesondere C, Si, $\mathrm{Mn}, \mathrm{P}, \mathrm{S}, \mathrm{N}$ etc.

- des Gefüges oder

- bruchmechanischer Kenngrößen wie $\mathrm{K}_{\mathrm{Ic}}, \mathrm{J}_{\mathrm{Ic}}, \mathrm{C}$, m oder $\Delta \mathrm{K}_{\text {th }}$.

In Phase I des Beurteilungsschemas wird empfohlen, Informationen zum Material aus alten Bemessungsvorschriften oder alten Lieferstandards für Baustahl $\mathrm{zu}$ entnehmen.

Falls in Phase I keine ausreichende Sicherheit nachgewiesen werden kann, sind vom beurteilenden Ingenieur in Phase II genauere, statistisch abgesicherte Materialda- 
ten für alte Stähle z. B. aus [1] zu verwenden. Nur wenn auch in dieser Phase noch keine ausreichende Sicherheit nachgewiesen werden kann, sollte in Phase III über zerstörende Probeentnahmen am Tragwerk und spezifische Materialuntersuchungen mit Hilfe von Kleinteilversuchen nachgedacht werden. Hierbei ist $\mathrm{zu}$ beachten, dass die Querschnitte, aus denen die Proben entnommen werden, nicht nennenswert geschwächt werden oder aber nicht ausgelastet sind. Wenn möglich, sollten nur kleine Proben aus gering beanspruchten Bereichen von Bauteilen entnommen werden. Es wird empfohlen, zur Auswahl der Anzahl der Proben und der Entnahmeorte nur erfahrene Ingenieure zu beauftragen.

In Bild 2 sind mögliche Entnahmeorte und eine mögliche Aufteilung von Proben dargestellt, die z. B. aus einem Stahltragwerk mit Hilfe einer Magnetbohrmaschine herausgebohrt wurden. Der ideale Probestückdurchmesser ist $\geq 60 \mathrm{~mm}$, wobei in besonderen Fällen auch kleinere Abmessungen möglich sind.

Falls die Probestücke lang genug sind, können schmale Kleinteilproben entnommen werden, die zumindest noch an einer Seite die Originalwalzoberfläche aufweisen und die als Flachproben für Zugversuche nach EN 10002 genutzt werden können. Probenahmen mit Hilfe von Stahlsägen sind ebenfalls möglich. Da Brennschneiden zu Aufhärtungen führen kann und die Wärmeeinflusszonen ggf. auch noch mit Zonen zusammenfallen, in denen eine erhöhte Konzentration von schädlichen Begleitelementen wie Schwefel und Phosphor vorhanden ist, ist eine Probenahme mit diesem Trennverfahren an alten Tragwerken zu vermeiden.

Bei der Entnahme wird weiter empfohlen, die Anforderungen gemäß EN 10025-1 zu beachten. Werden Materialdaten von Walzträgern ausgewertet, die an Proben aus dem Steg gewonnen wurden, ist zu berücksichtigen, dass die Festigkeit, Zähigkeit und insbesondere die chemische Zusammensetzung nennenswert von den Werten in den Flanschen abweichen können. Die Streckgrenze und Zugfestigkeit können im Steg deutlich höher, die Zähigkeit niedriger und der Anteil an schädlichen Begleitelementen aufgrund von Seigerungen höher sein als in den Flan- schen. Der kleinste Durchmesser einer standardisierten Rundprobe für einen Zugversuch sollte $5 \mathrm{~mm}$ nicht unterschreiten.

Das Material in alten genieteten Tragwerken unterscheidet sich wesentlich von modernem Stahl. Die Empfehlungen [1] erläutern ausführlich die Unterschiede zwischen den verschiedenen alten metallischen Werkstoffen und geben Hinweise, wie das Material eindeutig identifiziert werden kann. Es sind hierbei nach [14] Puddel- und Flussstähle als die wesentlichen Konstruktionswerkstoffe älterer Brücken zu unterscheiden.

Das 1784 von Henry Cort patentierte Puddelverfahrhen kann als das erste industrielle Stahlherstellungsverfahren betrachtet werden. Im Puddelofen wird der Verbrennungsraum für Steinkohle von dem Arbeitsherd für den Roheiseneinsatz durch eine Feuerbrücke getrennt. Die Erschmelzung und der Frischprozess, also die Oxidation des Kohlenstoffs, erfolgen durch die über den Arbeitsraum streichenden Verbrennungsgase. Die Oxidation der Schmelze wird durch die Schlackebildung behindert, so dass das Schmelzbad während des Frischvorgangs mehrfach umgerührt (engl. to puddle) werden muss. Mit abnehmendem Kohlenstoffgehalt und damit steigender Liquidustemperatur wird der Erstarrungsprozess bereits im Ofen eingeleitet, so dass ein Teil der untergerührten Schlacke nicht mehr an die Oberfläche steigen kann. Bei der anschließenden Herstellung und Weiterverarbeitung sogenannter Luppen im „teigigen“ Zustand wird ein Teil der Schlacke ausgetrieben. Insgesamt verbleibt jedoch eine so große Menge Schlacke im gewalzten Endprodukt, dass nach heutigen Maßstäben Puddelstahl nahezu als „Verbundwerkstoff" bezeichnet werden könnte. Das Gefüge ist daher durch einen schichtenförmigen Aufbau charakterisiert, der mineralische Einschlüsse enthält. Mit Hilfe eines Baumann-Abdruckes können die Schlackezeilen mit ihrem erhöhten Schwefelgehalt gut sichtbar gemacht werden. Der lagenförmige Aufbau des Puddelstahls ist auch der Grund für dessen anisotropes Verhalten, u. a. mit einer geringeren Festigkeit und Zähigkeit senkrecht zur Walzrichtung. Der Kohlenstoffanteil ist üblicherweise gering. Die chemische Analyse muss als Nassanalyse durchge-

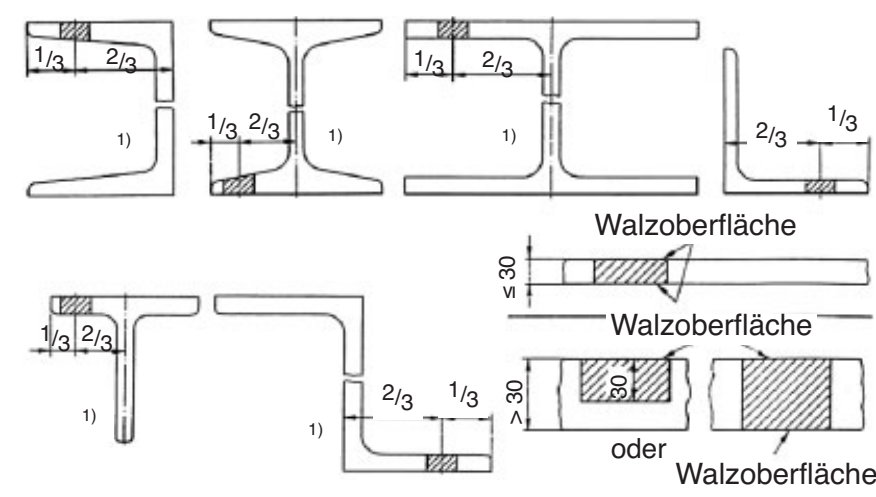

Probeentnahmeorte bei Walzquerschnitten nach EN 10025

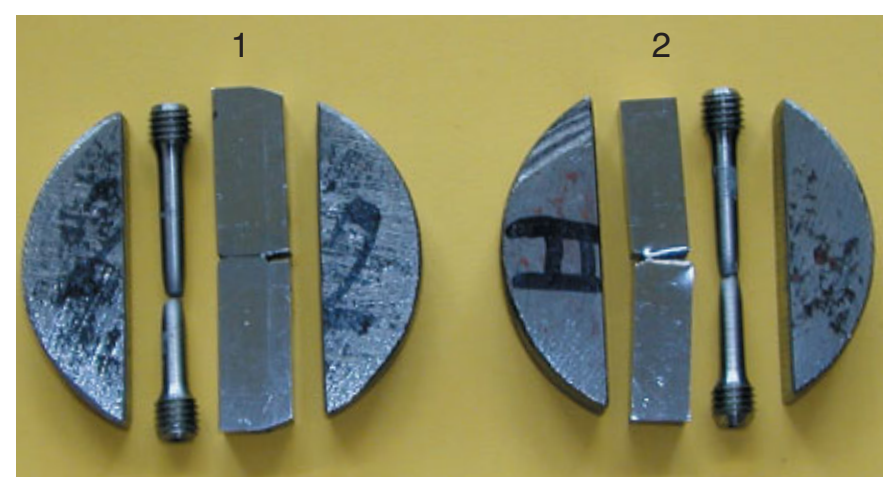

Probe 1: Versuchsstücke für den Baumannabdruck, den Zugversuch (B5), den Risswachstumsversuch an einer modifizierten SENB3-Probe und für die chemische Analyse

Probe 2: Versuchsstücke wie vorher aber mit Kerbschlagbiegeversuch anstelle SENB3-Probe

Bild 2. Probeentnahme aus Walzquerschnitten und Beispiele für die Anordnung von Versuchskörpern in durch Bohren mit Hohlbohrer gewonnene Rundproben

Fig. 2. Sampling in rolled cross sections and example for distribution of test specimens in the drilled sample 
führt werden, da das Funkenerosionsverfahren wegen der Inhomogenitäten des Werkstoffes nicht angewendet werden kann. Bild 3 zeigt einige der beschriebenen Charakteristika.

Die Möglichkeiten der Produktions- und Qualitätssteigerungen mit Puddelstahl zur Befriedigung der schnell steigenden Stahlnachfrage waren gering, so dass ab 1860 neu entwickelte Methoden der Flussstahlerzeugung den Puddelstahl sukzessive ablösten.

Die Flussstahlerzeugung begann mit dem 1850 von Henry Bessemer entwickelten gleichnamigen Verfahren, gefolgt vom Siemens-Martin-Verfahren (1865) und dem Thomas-Verfahren (1878). Bessemer- und Thomas-Verfahren waren Windfrischverfahren, bei denen der Frischvorgang durch Einblasen von Luftsauerstoff durch den Pfannenboden ablief. Das Bessemer-Verfahren erlaubte aufgrund der „sauren“ Prozessmetallurgie ausschließlich die Verwendung von phosphorarmen Roheisen. Das ThomasVerfahren dagegen ermöglichte wegen der basischen Ausmauerung und einer basischen Schlacke die Entfernung des Phosphors und des Schwefels aus dem Roheisens. Das Siemens-Martin-Verfahren ist als Herdfrischverfahren einzuordnen. Die von $F$. und $W$. Siemens entwickelte Regenerativfeuerung ermöglichte eine effektive Prozessführung mit ausreichend hohen Temperaturen. Die notwendige Baddurchmischung erfolgte durch das prozess- bedingte Aufkochen der Schmelze. Das Siemens-MartinVerfahren eignete sich im Gegensatz zum Thomas- und Bessemer-Verfahren sehr gut für die Schrottverarbeitung, wodurch die Stahlproduktion durch Rückführung von Altstoffen rentabler wurde.

Die über diese verbesserten Herstellungsprozesse erreichbare Festigkeit und die Zähigkeit des deutlich homogeneren Flussstahls sind höher als beim Puddelstahl. Früher Flussstahl ist bereits in der Lage, lokale Dehnungen an der Stelle der höchsten Beanspruchung durch lokales Plastizieren zu reduzieren. Bild 4 zeigt einen Versuchskörper aus Flussstahl nach einem Zugversuch mit nennenswerter Einschnürung und einen typischen Baumann-Abdruck. Die Terminologie für Flussstahl unterscheidet sich je nach Details im Herstellungsprozess. Einheitliche Begriffe finden sich erst mit der Entwicklung und Fortschreitung der Normung nach dem 2. Weltkrieg, wobei heute der Frischprozess überwiegend durch Sauerstoffaufblasen (OBM) erfolgt.

Die nachfolgend aufgeführte Zusammenstellung von Materialdaten basiert auf Untersuchungen von Materialproben für Puddelstahl wie auch Flussstahl aus deutschen Brücken.

Eine europäische oder gar weltweite Auswertung von Materialdaten wird $u$. U. zu leicht differierenden Werten führen. Jedoch können die angegebenen Werte auch in

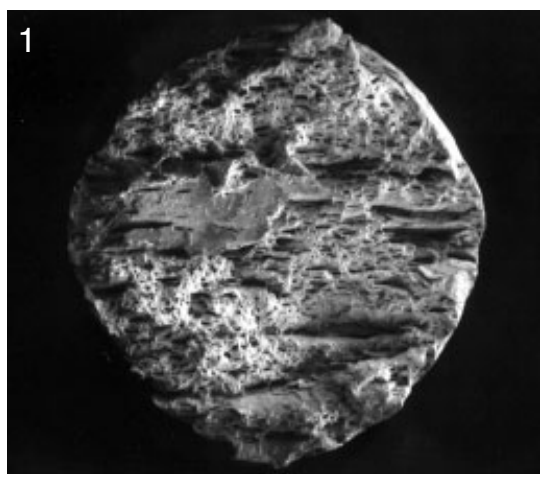

Rundprobe B5 nach einem Zugversuch (hier: Puddelstahl von 1890)

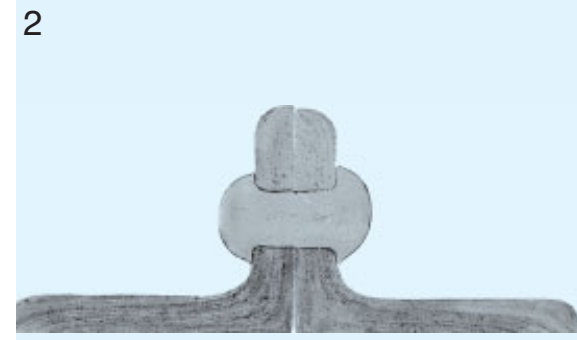

Baumann-Abdruck (hier: Puddelstahl von 1882 aus Deutschland)

Bild 3. Charakteristika von Puddelstahl

Fig. 3. Characteristics of puddled steel

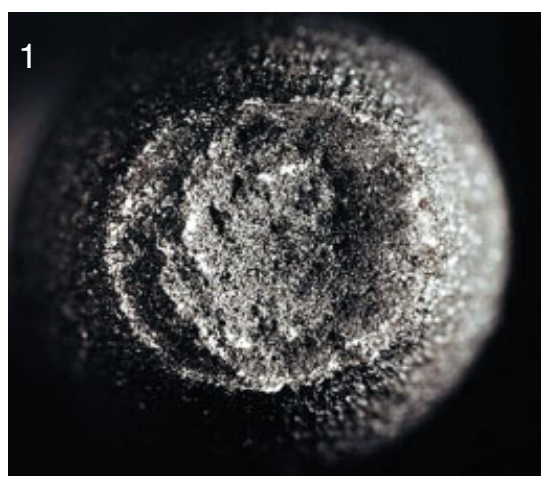

Rundprobe B5 nach einem Zugversuch (hier: Flussstahl von 1900)

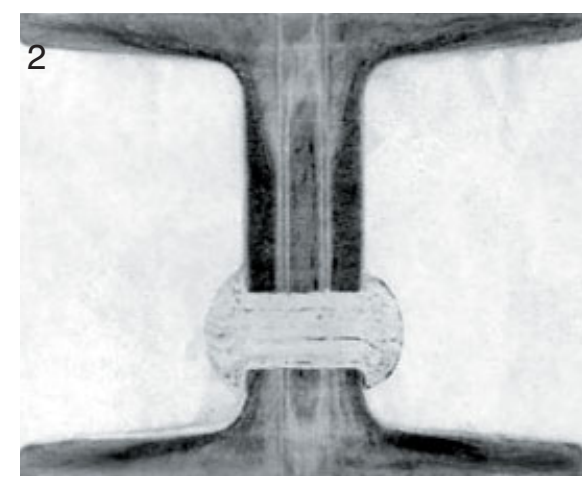

Baumann-Abdruck mit typischer Form der Steigerungen (hier: Flussstahl von 1900)

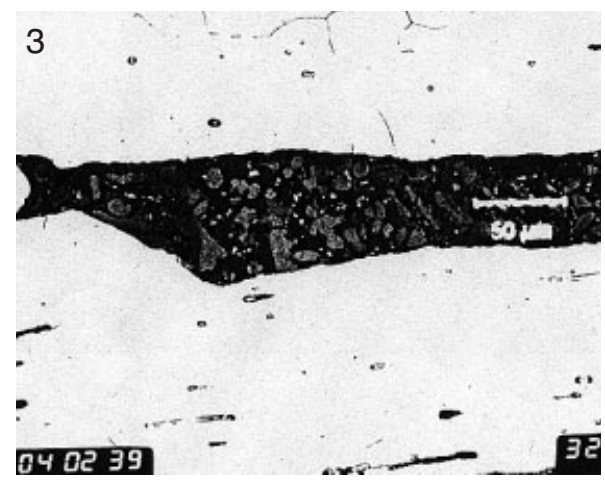

Gefüge mit großem linienförmigem Schlackeeinschluss

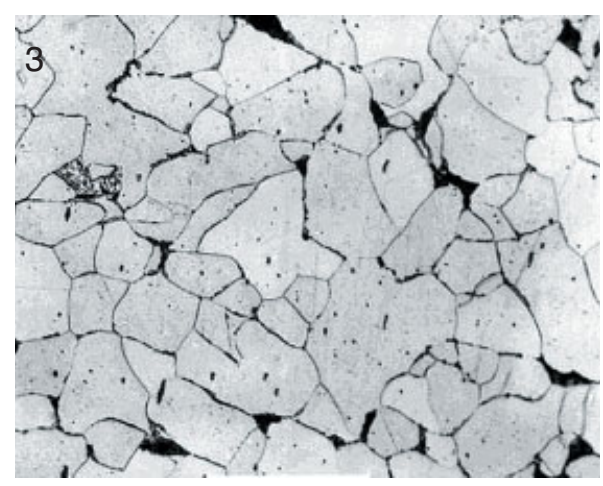

Gefüge mit großem linienförmigem Schlackeeinschluss

Bild 4. Charakteristika von frühem Flussstahl

Fig. 4. Characteristic of early mild iron (rimmed steel) 
anderen europäischen Ländern als Anhaltswerte verwendet werden.

Es ist zudem noch zu beachten, dass die Festigkeit mit abnehmender Temperatur zunimmt; bei den in Europa üblichen Tiefsttemperatur von $-30{ }^{\circ} \mathrm{C}$ und Baustählen S235 und S355 (früher St37 und St52) um rund 3,5 \%. Die in Tabelle 1 zusammengefassten Materialdaten sind, wenn nicht anders vermerkt, Mittelwerte und wurden bei $+10^{\circ} \mathrm{C}$ oder Raumtemperatur ermittelt.

Im Vergleich zu Puddelstahl ist das stabile Risswachstum im modernen Stahl langsamer und der Schwellenwert des zyklischen Spannungsintensitätsfaktors $\Delta \mathrm{K}_{\text {th }}$, unterhalb dessen es zu keinem weiteren Risswachstum kommt, im modernen homogenen Stahl kleiner.

Typischerweise wurden Puddelstahl und Flussstahl durch Nieten oder Schrauben verbunden.

Folgende allgemeine Hinweise zu den alten Stählen sollten beachtet werden:

1. Alte Stähle (Puddelstahl und unberuhigter Flussstahl) sind häufig nicht schweißbar oder nur sehr bedingt schweißbar. Puddelstahl sollte wegen seiner anisotropen Struktur niemals geschweißt werden. Sind Instandsetzungsmaßnahmen mit Hilfe von Schweißungen an unberuhigtem Flussstahl wegen besonderer Randbedingungen unumgänglich, sind in jedem Fall gesonderte Untersuchungen zur Schweißbarkeit durchzuführen, s. [1].

2. Erst in Phase III des Beurteilungsschemas sollte über zerstörende Probenentnahmen und die Bestimmung bauwerksspezifischer Materialdaten nachgedacht werden, wohingegen in den Phasen I und II allein mit den statistisch abgesicherten Mindestwerten gearbeitet werden sollte.

3. Zur Ermittlung von bruchmechanischen Kenngrößen wird auf amerikanische und britische Versuchsnormen verwiesen mit dem Hinweis, dass im Zusammenhang mit alten Bauwerken die sogenannte „Ein-Proben-Methode“ angewendet werden sollte. Bei dieser sollten zunächst relativ hohe zyklische Beanspruchungen auf die entsprechenden Kleinteilproben (CT, SENB3 etc.) aufgebracht werden, um die Rissfortschrittskonstanten $\mathrm{C}$ und $\mathrm{m}$ für die Beschreibung des stabilen Risswachstums nach Paris zu bestimmen. Sind hierzu ausreichende Versuchsdaten gewonnen, kann die zyklische Beanspruchung stetig reduziert werden, um so im gleichen Versuch auch noch den Schwellenwert des zyklischen Spannungsintensitätsfaktors $\Delta \mathrm{K}_{\text {th }} \mathrm{zu}$ ermitteln. Wird dabei das Risswachstum kleiner als $10^{-8} \mathrm{~mm}$ pro Lastwechsel, ist der Schwellenwert $\Delta \mathrm{K}_{\text {th }}$ gemäß einer Definition nach Taylor erreicht. Nach der britischen Norm BSPD 6493 kann der Schwellenwert für alte Stähle wie folgt angenommen werden:

$$
\begin{aligned}
& \mathrm{R} \leq 0,5: \Delta \mathrm{K}_{\text {th }}(\mathrm{R})=170-214 \mathrm{R}\left[\mathrm{N} / \mathrm{mm}^{3 / 2}\right] \\
& \mathrm{R}>0,5: \Delta \mathrm{K}_{\mathrm{th}}=63\left[\mathrm{~N} / \mathrm{mm}^{3 / 2}\right]=2[\mathrm{MPa} \sqrt{\mathrm{m}}]
\end{aligned}
$$

4. Es wird darauf hingewiesen, dass entsprechende Versuche in Deutschland und in der Schweiz auch schon Schwellenwerte des Risswachstums ergeben haben, die über $100 \%$ größer sind als die vorgenannten Werte gemäß der britischen Norm. Da dieser Wert die Abschätzung der $\mathrm{zu}$ erwartenden Restnutzungsdauer und die sicheren Be-

Tabelle 1. Mechanische und chemische Eigenschaften von Puddel- und Flussstahl basierend auf [9].

\begin{tabular}{|c|c|c|c|}
\hline \multirow{2}{*}{ Versuch und zugehörige Standards } & Puddelstahl & Flussstahl & \multirow{2}{*}{ Bemerkung } \\
\hline & $\mathrm{N} / \mathrm{mm}^{2}$ & $\mathrm{~N} / \mathrm{mm}^{2}$ & \\
\hline \multicolumn{2}{|l|}{ Zugversuch: EN 10002, Teil 1} & & Vorh. Versuche $(>600)$ \\
\hline Streckengrenze $\mathrm{R}_{\mathrm{eL}}$ & 203 & 229 & \multirow{3}{*}{$\begin{array}{c}\text { Zugproben: } \\
\text { Min. } \varnothing: 5 \text { mm (B5); } \\
\text { Min. Dicke: } 4 \text { mm (E4) }\end{array}$} \\
\hline Zugfestigkeit Rm & & & \\
\hline $\begin{array}{l}\text { Dehnung } \varepsilon, \min : \\
\text { max: }\end{array}$ & $\begin{array}{c}6,0 \\
26,5\end{array}$ & $\begin{array}{l}22 \\
42\end{array}$ & \\
\hline \multicolumn{4}{|l|}{ Rissfortschrittsversuche nach ASTM, BS } \\
\hline $\begin{array}{l}\text { Paris-Konstante } \mathrm{C} \text {, max. } \\
\text { min: }\end{array}$ & $\begin{array}{l}4,9 \cdot 10^{-17}(\mathrm{R}=0,1) \\
5,7 \cdot 10^{-28}(\mathrm{R}=0,5)\end{array}$ & $0,5739 \cdot 10^{-13}$ & \multirow{3}{*}{$\begin{array}{c}\text { Probenform: } \\
\text { SENB3, CT, } \\
\text { modifizierte CT } \\
\text { Empfehlung: } \\
\mathrm{K}_{\mathrm{th}}=2 \mathrm{MPa} \mathrm{m} \mathrm{M}^{1 / 2} \\
\mathrm{C}=4 \cdot 10^{-13}, \mathrm{~m}=3\end{array}$} \\
\hline $\begin{array}{l}\text { Paris-Konstante m, max. } \\
\text { min: }\end{array}$ & $\begin{array}{l}9,3(\mathrm{R}=0,5) \\
3,8(\mathrm{R}=0,3)\end{array}$ & 3,299 & \\
\hline $\begin{array}{l}\text { Schwellenwert des zyklischen Spannungs- } \\
\quad \text { intensitätsfaktors } \Delta \mathrm{K}_{\text {th }}\left[\mathrm{N} / \mathrm{mm}^{3 / 2}\right]\end{array}$ & $\begin{array}{c}13,49(\mathrm{R}=0,1) \\
6,36(\mathrm{R}=0,5)\end{array}$ & $6,2(\mathrm{R}=0,3)$ & \\
\hline \multicolumn{3}{|l|}{ Chemische Analyse } & \multirow{7}{*}{$\begin{array}{l}\text { 1) Nur Nassanalyse möglich } \\
\text { 2) wenn der Stickstoffgehalt } \\
\text { kleiner ist als ca. } 0,014 \% \\
\text { können Alterungseffekte } \\
\text { vernachlässigt werden. }\end{array}$} \\
\hline $\mathrm{C}$ & $0,0032-0,15$ & $0,026-0,20$ & \\
\hline $\mathrm{Si}$ & $0,003-0,42$ & $0,001-0,013$ & \\
\hline $\mathrm{S}$ & $0,0034-0,018$ & $0,063-0,176$ & \\
\hline $\mathrm{P}$ & $0,011-0,39$ & $0,009-0,136$ & \\
\hline $\mathrm{N}$ & $0,0037-0,04^{2)}$ & $0,011-0,022$ & \\
\hline Mn & $0,054-0,11$ & $0,036-0,52$ & \\
\hline
\end{tabular}

Table 1. Mechanical and chemical properties of puddled steel and mild steel (rimmed steel), based on test results [9] 
triebszeitintervalle deutlich vergrößern kann, wird empfohlen, bei Materialuntersuchungen in Phase III besonders diese Materialkenngrößen $\mathrm{zu}$ untersuchen und $\mathrm{zu}$ verifizieren.

5. Werden Materialuntersuchungen notwendig, so gilt folgendes Prinzip für die zerstörende Probeentnahme:

Probestücke so groß wie für die Materialuntersuchungen nötig, aber so klein wie möglich.

6. Keine Schweißungen oder Brennschnitte an Bauteilen aus Puddelstahl oder Flussstahl, wenn nicht eindeutig nachgewiesen wurde, dass das Material mindestens bedingt schweißgeeignet ist.

7. Kerbschlagbiegeversuche an alten Stählen sind aufgrund der spezifischen Gefügestruktur nicht aussagekräftig. Es ist bekannt, dass der in diesen Versuchen ermittelte Übergangsbereich von der Tieflage mit überwiegend sprödem Werkstoffverhalten zur Hochlage mit überwiegend zähem Werkstoffverhalten bei alten Stählen häufig bei $+10^{\circ} \mathrm{C}$ oder noch höher liegt, die gleichen Stähle in Bruchmechanikversuchen aber eine ausreichende Werkstoffzähigkeit aufweisen. Die zu bevorzugende bruchmechanische Kenngröße zur Charakterisierung der vorhandenen Zähigkeit alter Stähle sollte deshalb entweder die direkt gemessene oder die aus dem kritischen Spannungsintensitätsfaktor $\mathrm{K}_{\mathrm{Ic}}$ umgerechnete kritische Größe des J-Integrals $\left(\mathrm{J}_{\mathrm{Ic}}\right)$ sein.

Dem Nachweis der Schweißbarkeit ist aufgrund der Wichtigkeit ein eigenes Kapitel in [1] gewidmet. Hier wird aufgrund des Umfangs der dazu notwendigen Informationen nicht weiter darauf eingegangen. Eine andere Quelle zu diesem Thema ist [10].

\subsection{Ermüdungsfestigkeitskurve}

In Phase I des vorgeschlagenen Beurteilungsschemas beziehen sich die Abschätzungen der noch verbleibenden Restlebensdauer auf Ermüdungsfestigkeitskurven (Wöhlerlinien). Die in Bild 5 dargestellte S-N-Kurve hat sich in der Vergangenheit für konservative Abschätzungen der Restle- bensdauer von genieteten Tragwerken durchgesetzt. Zur Absicherung dieser Kurve wurden in [11] und [12] eine Vielzahl von Großbauteilversuchen, u. a. auch an Originalbrückenträgern gesammelt und streng nach den statistischen Auswerteverfahren nach Eurocode 3 ausgewertet. Dabei zeigte sich, dass die nachfolgend dargestellte Wöhler-Kurve entsprechend Kerbklasse 71 für alle genieteten Bauteile als sicher angesehen werden kann. Es wird darauf hingewiesen, dass bedingt durch ein relativ schnelleres Risswachstum in alten Stählen verglichen mit modernen Stählen bei höherem Schwellenwert für spezifische Bauteilgeometrien insbesondere bei aus vielen Profilen zusammengesetzten Querschnitten und bei bestimmten von dem jeweiligen Bauteilaufbau abhängigen Beanspruchungsarten deutlich günstigere Kerbfalleinstufungen bis zu Kerbklasse 90 möglich sind. Zusammengesetzte Profile zeichnen sich dadurch aus, dass der Riss im benachbarten Element neu initiiert werden muss. Liegen solche Bauteile vor und sollen die angesprochenen Reserven z. B. in Phase III des Beurteilungsschemas ausgenutzt werden, ist die Wahl der günstigeren Kerbfallklasse für den Einzelfall detailliert zu begründen und mit der zuständigen Bauaufsichtsbehörde dessen Verwendung gesondert abzustimmen.

Für die Ermüdungsbewertung einer bestehenden geschweißten Stahlstruktur sollen die Konstruktionsdetails und Kerbfallklassen von Eurocode 3, Teil 1-9 [13] angewendet werden.

\section{Ermittlung von Bauwerksinformationen durch Inspektion und Prüfung 4.1 Allgemein}

Die Empfehlungen [1] beschränken sich ausschließlich auf das Ermüdungsverhalten von bestehenden Stahlkonstruktionen, insbesondere von Stahlbrücken, sowie auf daraus resultierende Maßnahmen in Form von Inspektionen und Ermittlungen der Bauwerksabmessungen, mit dem Ziel, eine trotz Materialermüdung sichere Weiternutzung der bestehenden Konstruktion zu gewährleisten.

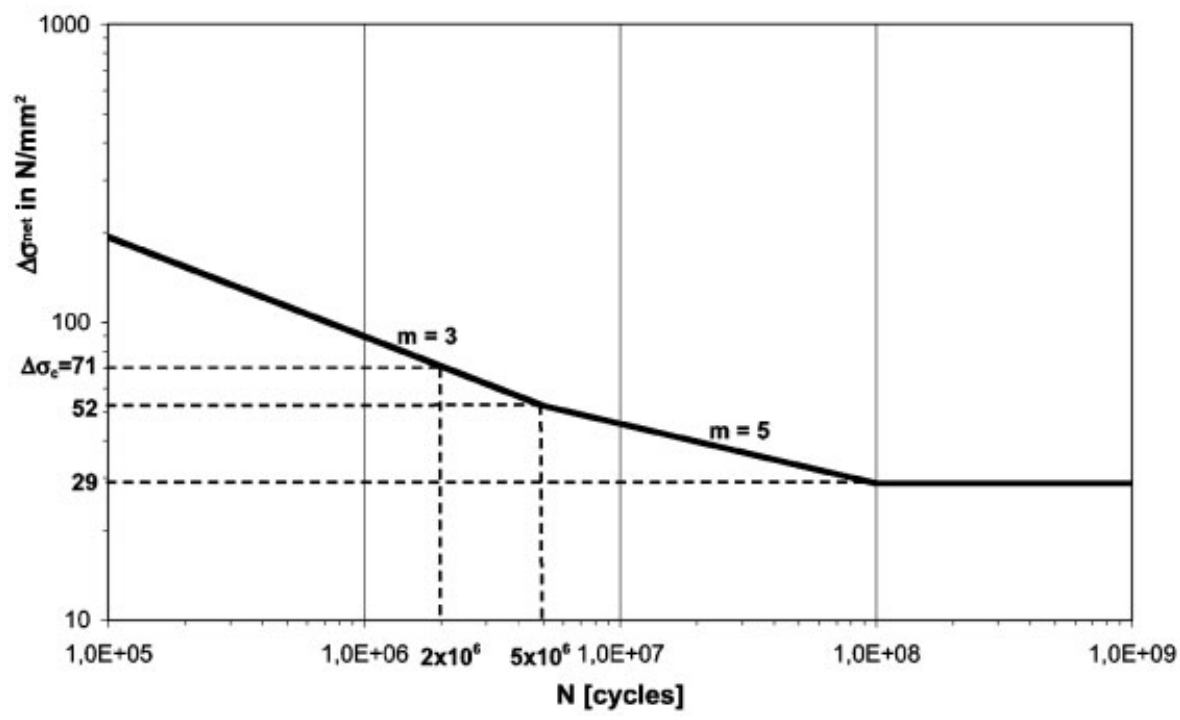

Kerbklasse: $71 \mathrm{~N} / \mathrm{mm}^{2}$, Dauerfestigkeit: $52 \mathrm{~N} / \mathrm{mm}^{2}$, Schwellenwert der Ermüdungsfestigkeit: 29 N/mm²

Bild 5. Empfohlene Wöhlerlinie (S-N-Kurve) zur Beurteilung genieteter Stahltragwerke

Fig. 5. S/N-curve proposed for fatigue assessment of riveted structures 
Je älter die Brücken oder die Konstruktionen sind, um so mehr Teilschäden haben sie im Verlauf der Betriebsdauer akkumuliert, d. h., es ist mit ihnen zu rechnen im doppelten Sinn des Wortes.

Die im Rahmen von Bauwerksprüfungen und Inspektionen beobachteten Ermüdungsschäden in Form von Anrissen können im Allgemeinen nachfolgende unterschiedliche Entstehungsursachen haben:

- Herstellungs- und Fertigungsschäden infolge des Nietens oder Schweißens

- Schlechte Konstruktionsdetails mit großer Kerbwirkung

- unvorhergesehene Verformungen und daraus resultierende hohe sekundäre Spannungsamplituden während des Betriebs

- unzureichendes Wissen während der Planung und Ausführung.

Während der Hauptinspektion von Brücken mit visueller Prüfung alle sechs Jahre werden in der Sichtprüfung meist fast alle Schäden detektiert. Größere Schäden werden auch während der jährlichen visuellen Inspektion von unterhalb des Bauwerks oder während der i. A. alle drei Jahre zwischen den Hauptprüfungen durchgeführten Zwischeninspektion kritischer Details in der Regel entdeckt.

\subsection{Inspektionen und zerstörungsfreie Prüfverfahren}

Art, Umfang und Zeitintervall der durchzuführenden Inspektionen von Brücken bzw. Bauwerksprüfungen richten sich im Allgemeinen nach den jeweils national vorhandenen Richtlinien und Normen der Baulastträger (Straßenbau- oder Eisenbahnbehörde, Land oder Gemeinde). International üblich ist ein Inspektionsintervall von fünf bis sechs Jahren in Form von Sichtprüfungen. Für Straßenbrücken ist dies beispielsweise in Deutschland im Detail in DIN 1076:1999 geregelt. Neben den standardmäßig im Rahmen von Brückenprüfungen statt- findenden einfachen Sichtprüfungen wurden in den vergangenen Jahren im Bereich der zerstörungsfreien Prüfung (ZfP) Standardverfahren aus der Industrie auch für ihren Einsatz an Bauwerken weiterentwickelt, die derzeit vermehrt im Rahmen von objektbezogenen Schadensanalysen eingesetzt werden.

Tabelle 2 enthält eine Zusammenstellung aktuell angewandter zerstörungsfreier Prüfverfahren. Nähere Informationen inklusive Anwendungsbereiche sowie Vor- und Nachteile sind in ausführlicher Form in [1] enthalten.

\subsection{Maßnahmen zur Ermittlung des Bauwerkszustands (Bestandsaufnahme)}

Gelingt es nicht im Rahmen von Phase I oder II des Beurteilungsschemas, gemäß Bild 1, den Nachweis ausreichender Sicherheit gegen Ermüdungsversagen $\mathrm{zu}$ führen, so sind weitergehende Untersuchungsmaßnahmen zu ergreifen, um den vorhandenen Bauwerkszustand möglichst detailliert beschreiben zu können und damit Unsicherheiten der in den Phasen I und II getroffenen Annahmen auszuräumen. Der Ermüdungsnachweis ist dann, entsprechend Bild 1, in der Phase III unter Hinzuziehung von Experten zu führen.

Im Hinblick auf die Beurteilung der Ermüdungssicherheit sind mögliche weitergehende Untersuchungsmaßnahmen $u$. a. unter folgenden Gesichtspunkten durchzuführen:

- Ermittlung der Bauwerksgeometrie und der Materialparameter

- Beurteilung der gewählten statischen und ggf. dynamischen Modellbildung

- Beurteilung der vorliegenden bzw. vergangenen Belastungssituation (Verkehrslasten)

- Ermittlung der ermüdungsrelevanten Spannungskenngrößen und Sekundärspannungen

Tabelle 2. Zerstörungsfreie Prüfmethoden zur Feststellung eventuell vorhandener Ermüdungsschäden

Table 2. Non-Destructive inspection tools for detection of fatigue damages

\begin{tabular}{|c|c|c|}
\hline Methode & Auffindbare Rissart & Bemerkungen \\
\hline Sichtprüfung & Oberflächenrisse & $\begin{array}{c}\text { Mit Hilfe von Vergrößerungsgläsern } \\
\text { und entsprechender Erfahrung: } \\
\text { gute Ergebnisse und Zuverlässigkeit }\end{array}$ \\
\hline Magnetpulverprüfung & Oberflächenrisse & Nur für magnetisches Material \\
\hline Farbeindringverfahren & Oberflächenrisse & $\begin{array}{c}\text { Auch für nicht magnetisches Material } \\
\text { geeignet }\end{array}$ \\
\hline Durchstrahlungsprüfung & $\begin{array}{c}\text { Oberflächenrisse und Riss im Bauteil- } \\
\text { inneren oder in verdeckten Profilen }\end{array}$ & $\begin{array}{c}\text { Spezielle Prüfer mit spezieller Aus- } \\
\text { bildung und Zulassung erforderlich; } \\
\text { bestimmte Schutzbereiche sind } \\
\text { abzusperren }\end{array}$ \\
\hline Ultraschallprüfung & $\begin{array}{c}\text { Oberflächenrisse und Riss im } \\
\text { Bauteilinneren, wobei nur unverdeckt } \\
\text { liegende Profile untersucht werden können }\end{array}$ & $\begin{array}{c}\text { Nicht anwendbar bei Schweißeisen } \\
\text { Riss an Nietlochwandungen }\end{array}$ \\
\hline Wirbelstromprüfung & Bisher nur unter Laborbedingungen \\
erprobt
\end{tabular}


Tabelle 3 gibt einen Überblick über derartige weitergehende Untersuchungsmaßnahmen und hierfür geeignete Messverfahren.

Die in Tabelle 3 aufgeführten Messverfahren zur Ermittlung des Bauwerkszustandes können entweder in Form von diskreten Einzelmessungen oder als eine fortlaufende messtechnische Aufzeichnung und Überwachung (Bauwerksmonitoring) angewandt werden. Im Bereich der Materialermüdung wird das Bauwerksmonitoring derzeit vor allem zur Ermittlung der für die Schädigungsberechnung erforderlichen Beanspruchungskollektive - verursacht durch die Verkehrsbelastung - verwendet oder auch zur Ermittlung zeitabhängiger Änderungen allgemeiner Bauwerksparameter, wie z. B. Verformungen.

Für die Bewertung der Ermüdungssicherheit werden im Rahmen von Bauwerksüberprüfungen vielfach nachfolgende Messungen und Kontrollen durchgeführt, die zum Ziel haben, die aus dem tatsächlichen Tragverhalten resultierenden ermüdungsrelevanten Spannungsschwingbreiten möglichst genau und detailliert zu erfassen:

- Ermittlung des Dehnungs- und Spannungszustands in den hoch beanspruchten Tragwerksteilen

- Überprüfung der Wirkungsweise spezieller Tragwerkskomponenten, z. B. der Bremsverbände

- Ermittlung der Dehnungs- bzw. Spannungsnulllinie in einzelnen Querschnitten

- Untersuchung von Details, die sich in anderen Bauwerksabschnitten als ermüdungsgefährdet erwiesen haben
- Ermittlung sekundärer i.d. R. verformungsinduzierter Spannungskomponenten

- Ermittlung von tatsächlichen Einspann- und Auflagermomenten

- Überprüfung der Funktionsfähigkeit von Lagern und Fahrbahnübergängen

- Überprüfung von Dehnungen bzw. Spannungen in theoretisch unbeanspruchten Tragwerksteilen, z. B. in Nullstäben bei Fachwerkkonstruktionen

- Ermittlung von Dehnungs- und Spannungskonzentrationen im Bereich von Verbindungen und Gelenken.

\section{Maßnahmen zur Weiternutzung bestehender Konstruktionen}

Gemäß des in Bild 1 aufgeführten Beurteilungsschemas zur Bewertung der Ermüdungssicherheit bestehender Konstruktionen ist am Ende, in Phase IV, eine abschließende Entscheidung über die mögliche Weiterverwendung oder den Ersatz der Konstruktion zu treffen. In Abhängigkeit der Ergebnisse der vorangegangen Phasen I bis III ist eine Weiternutzung der bestehenden Konstruktion prinzipiell durch folgende Maßnahmen möglich:

- Reduzierung der Verkehrsbelastung hinsichtlich Größe und/oder Intensität (Verkehrsvolumen)

- Reparatur und Sanierung von bestehenden Schäden

- Verstärkung und Ertüchtigung des Tragwerks.

Bei der Planung, Berechnung und Ausführung von Reparatur- und Verstärkungsmaßnahmen muss sehr sorgfältig

Tabelle 3. Ziel der Messungen und dabei zu bevorzugende Messaufnehmer

Table 3. Measurement tasks and preferred sensors

\begin{tabular}{|c|c|c|}
\hline Untersuchungsgegenstand & Messverfahren & Kommentar / Genauigkeit \\
\hline Geometrieaufnahme & $\begin{array}{l}\text { Handaufmaß, Fotogrammetrie, } \\
\text { Laser-Scan-Verfahren }\end{array}$ & $\begin{array}{c}\approx 1 \mathrm{~mm} \\
\approx 1 \mathrm{~mm} \text { bis } 0,1 \mathrm{~mm} \text { je nach } \\
\text { Objektiventfernung }\end{array}$ \\
\hline Materialparameter & $\begin{array}{l}\text { Zerstörende Prüfungen an } \\
\text { Bohrkernen, z.B. Zugversuch, } \\
\text { Kerbschlagbiegeversuch, ... }\end{array}$ & vgl. Bild 2 \\
\hline $\begin{array}{l}\text { Lokaler Dehnungs- und daraus } \\
\text { folgender Spannungszustand }\end{array}$ & $\begin{array}{c}\text { Dehnungsmessstreifen } \\
\text { Faseroptische Dehnungssensoren }\end{array}$ & $\begin{array}{c}\approx 0,1 \mathrm{~mm} / \mathrm{m} \\
\text { Genauigkeit abhängig vom Sensor }\end{array}$ \\
\hline $\begin{array}{l}\text { Statisches Tragverhalten und } \\
\text { statisches System/Modell }\end{array}$ & System von Dehnungsmessstreifen & $\approx 0,1 \mathrm{~mm} / \mathrm{m}$ \\
\hline $\begin{array}{c}\text { Dynamisches Tragverhalten: } \\
\text { - Beschleunigungen } \\
\text { - Eigenfrequenzen }\end{array}$ & $\begin{array}{l}\text { Beschleunigungsmesser, -sensoren } \\
\text { Dehnungsaufnehmer }\end{array}$ & $\approx 0,05$ bis $1 \%$ je nach Kraftgröße \\
\hline Temperatur & Thermoelemente, -sensoren & $\begin{array}{l}\approx 0,1 \mathrm{~K} \text { je nach max. } \\
\text { Temperaturschwankung }\end{array}$ \\
\hline $\begin{array}{l}\text { Neigungsmessung, } \\
\text { Auflagerverdrehung }\end{array}$ & $\begin{array}{l}\text { Neigungsmesser, } \\
\text { Rotationswegaufnehmer, } \\
\text { Inklinomenter }\end{array}$ & $\approx 0,001 \mathrm{bis} 0,01^{\circ}$ \\
\hline $\begin{array}{l}\text { Verformungen, } \\
\text { Auflagersetzungen }\end{array}$ & $\begin{array}{l}\text { Induktiver Wegaufnehmen } \\
\text { Laser-Scan-Verfahren }\end{array}$ & $\begin{array}{l}\approx 0,1 \% \text { der max. Verformung } \\
\approx 1 \mathrm{~mm} \text { bis } 0,1 \mathrm{~mm} \text { je nach } \\
\text { Objektiventfernung }\end{array}$ \\
\hline Verkehrsbelastung & $\begin{array}{c}\text { Hydrauliksenoren zur } \\
\text { Datenerfassung von bewegter Masse } \\
\text { (Weigh-in-Motion- oder kurz } \\
\text { WIM-Systeme genannt) }\end{array}$ & \\
\hline
\end{tabular}


vorgegangen werden, da Änderungen an einem vorhandenen System und in ermüdungskritischen Tragwerksbereichen immer auch Auswirkungen auf benachbarte Tragwerksbereiche haben können und somit eine mögliche neue Ermüdungsgefährdung verursacht werden kann. Aus diesem Grund enthalten die Empfehlungen [1] eine auf Niet- und Schweißkonstruktionen abgestimmte tabellenförmige Zusammenstellung von typischen bei Stahlbrücken beobachteten Schadensbildern inklusive $\mathrm{zu}-$ gehöriger Reparatur- und Ertüchtigungsvorschläge. Diese Zusammenstellung basiert $u$. a. auf einer Dokumentation des International Institute of Welding (IIW), Working Group 5 [8] für geschweißte Konstruktionen und auf umfangreichen Ermüdungsversuchen in der Bundesanstalt für Materialforschung und -prüfung [9] für genietete Konstruktionen. Ergänzungen zu dieser Sammlung aus neuern Erfahrungen aus der Ingenieurpraxis wären wünschenswert.

\section{Anwendungsbeispiel}

Die Empfehlungen [1] enthalten zum Schluss noch ein Anwendungsbeispiel, mit dessen Hilfe die generelle Vorgehensweise erläutert wird.

In dem Beispiel wird eine genietete einfeldige Fachwerkbrücke untersucht. Im Jahr 2000 hatte diese Brücke nach über 100 Jahren Benutzung theoretisch das Ende ihrer Lebensdauer erreicht. Im Beispiel werden die Berechnungen für die Diagonale eines Hauptträgers durchgeführt, für die in Phase I der Untersuchung abgeschätzt wurde, dass dieses Tragelement für eine Ermüdungsbeurteilung kritisch sein könnte. Die vorgestellten Abschätzungen basieren auf Informationen über die Zuganzahl und transportierten Tonnen in der Vergangenheit und zeigen mit Hilfe einer Schadensakkumulationsberechnung, dass keine rechnerische Restlebensdauer mehr vorhanden ist. Aufgrund der Tatsache, dass dieses Bauteil in der vorgeführten Analyse als nicht mehr sicher einzustufen ist, sind weitere Maßnahmen erforderlich, die ebenfalls im Beispiel vorgestellt werden.

\section{Zusammenfassung}

Mit der Veröffentlichung der Empfehlungen [1] wurde ein phasenweises Bewertungsverfahren für bestehende ermüdungsbeanspruchte Stahltragwerke vorgestellt, welches als Grundlage für eine zukünftige europaweit einheitliche Normenregelung von der Kommission angenommen wurde. Die Empfehlungen sind so aufbereitet, dass sie von baupraktisch tätigen Ingenieuren für die Beurteilung alter Stahltragwerke verwendet werden können. Das vorgeschlagene Beurteilungsschema ist in vier Phasen unterteilt. $\mathrm{Zu}$ jeder dieser Phasen wird empfehlend darauf hingewiesen, welche Art von Fachleuten, ob und wenn ja welche zerstörungsfreien Prüfmethoden, ob und wenn ja welche Messungen am Tragwerk und ob Materialuntersuchung zur Beurteilung eines bestehenden Tragwerkes durchgeführt bzw. hinzugezogen werden sollten. Die Empfehlungen sind so geschrieben, dass die in der Brückenbaupraxis tätigen Ingenieure/innen ohne nennenswerte Erfahrung in der Beurteilung bestehender Tragwerke die ersten beiden Phasen weitgehend alleine durchführen können. Am Ende jeder Phase muss der jeweilige Baulastträger über die erzielten Ergebnisse schriftlich informiert werden, wobei die Ergebnisse so aufzubereiten und aus technischer Sicht zu bewerten sind, dass die Entscheidungen zum weiteren Vorgehen einfach vorzunehmen sind.

\section{Danksagung}

An dieser Stelle möchten die Autoren die Gelegenheit nutzen, den an der Entstehung der Empfehlung Beteiligten herzlich zu danken. Dies sind zum einen die beiden Organisationen EKS und JRC, unter deren Dach die Arbeiten und die Veröffentlichung durchgeführt wurden. Zum anderen sei nachfolgend Genannten ganz herzlich für die Mitarbeit und die Unterstützung gedankt: Mladen Lukic, Henk Kolstein, Scott Walbridge, Boris Androic, Onno Dijkstra und Ömer Bucak (übrige Autoren von [1]) sowie Gerhard Sedlacek, Frans Bijlaard, Michel Géradin, António Pinto und Silvia Dimova (Herausgeber). Abschließend möchten wir uns im Namen aller Beteiligten noch ganz herzlich bei Klaus Brandes, Winfried Dahl, Markus Feldmann, John W. Fisher, Haig Gulvanessian, H.M.C.M. van Maarschalkerwaart, Gerhard Sedlacek, Natalie Stranghöner, Robert Sweeney und Marcel Tschumi bedanken, die die Empfehlungen in technischer und textlicher Hinsicht Korrektur gelesen haben. Für die Übernahme des Lektorats des vorliegenden Artikels danken wir Eckhard Kühn.

\section{Literaturhinweise}

[1] Kühn, B., Lukic, M., Nussbaumer, A., Günther, H.-P., Helmerich, R., Herion, S., Kolstein, M.H., Walbridge, S., Androic, B., Dijkstra, O., Bucak, Ö.: Assessment of Existing Steel Structures: Recommendations for Estimation of Remaining Fatigue Life. JRC Scientific and Technical reports, Joint Report Prepared under the JRC - ECCS cooperation agreement for the evolution of Eurocode 3 (programme of CEN/TC 250). Editors: G. Sedlacek, F. Bijlaard, M. Géradin, A. Pinto and S. Dimova. First Edition, February 2008, EUR 23252 EN, ISSN 1018-5593, JRC 43401

(http://eurocodes.jrc.ec.europa.eu).

[2] JCSS, Joint Committee on Structural Safety: Probabilistic assessment of existing structures. Rilem publication, 2001.

[3] Crocetti, R.: Constant Amplitude Fatigue Limit for riveted girders. Acta Polytecnica-Eurosteel 1999, Prague Vol. 39, 5/1999.

[4] Miner, M.: Cumulative damage in Fatigue. Journal of Applied Mechanics, pp. A159-A164, 1945.

[5] Hensen, W.: Grundlagen für die Beurteilung der Weiterverwendung alter Stahlbrücken. Dissertation, RWTH Aachen, Lehrstuhl für Stahlbau, Heft 21, 1992.

[6] Kunz, P.: Probabilistisches Verfahren zur Beurteilung der Ermüdungssicherheit bestehender Brücken aus Stahl. Dissertation No1023, EPF Lausanne, 1992.

[7] Eurocode 3: Design of steel structures, Part 1-9 Fatigue, CEN, Brussels, 2005 (Final version).

[8] WG5, IIW, website http://iiw-wg5.cv.titech.ac.jp/default.htm, WG5 Leader: Prof Miki, Japan.

[9] Helmerich, R.: Alte Stähle und Stahlkonstruktionen - Materialuntersuchungen, Ermüdungsversuche an originalen Brückenträgern und Messungen von 1990 bis 2003. BAM, Forschungsbericht 271, Berlin, 2005.

[10] Lüddecke, F.: Ein Beitrag zur Ertüchtigung bestehender Stahltragwerke unter besonderer Berücksichtigung des Fügeverfahrens Schweißen. BAM Dissertationsreihe Band 17. 
Bremerhaven: Wirtschaftsverlag N.W., Verlag für neue Wissenschaft GmbH 2007.

[11] ORE DT 176 (D154): Statistische Auswertung von Ermüdungsversuchen an Nietverbindungen in Flussstahl. Forschungs- und Versuchsamt des Internationalen Eisenbahnverbandes ORE, Utrecht, 1986.

[12] Muncke, M., Quoos, V., Tschumi, M., Sedlacek, G., Feldmann, M., Kühn, B., Brandes, K., Helmerich, R., Hirt, M., Nussbaumer, A., Bassetti, A., Geissler, K., Gut, J., Kunz, P., Liechti, P.: Vereinheitlichter Sicherheitsnachweis für bestehende Stahlbrücken. Endbericht im Rahmen der Zusammenarbeitsvereinbarung DB AG - SBB, 31. 05. 2001.

[13] DIN EN 1993-1-9:2005-07, Eurocode 3: Bemessung und Konstruktion von Stahlbauten, Teil 1-9: Ermüdung

[14] Langenberg, P.: Bruchmechanische Sicherheitsanalyse anrißgefärdeter Bauteile im Stahlbau. Diss., RWTH Aachen, Shaker Verlag, Band 14/95, 1995, ISBN 3-8265-1102-6
[15] Murakami, Y.: Stress Intensity Factors Handbook. Volume 1, Volume 2. Frankfurt/M.: Pergamon Press, 1987.

[16] Nussbaumer, A., Günther, H.-P.: Grundlagen und Erläuterung der neuen Ermüdungsnachweise nach Eurocode 3. Stahlbau Kalender, 2006, S. 381-484,hrsg. v. U. Kuhlmann. Berlin: Ernst und Sohn 2006.

\section{Autoren dieses Beitrages:}

Dr.-Ing. Bertram Kühn, Leiter der Abteilung Brückenbau bei Verheyen Ingenieure GmbH Co. KG, Wilhelmstraße 7 - 11, 55543 Bad Kreuznach, Dipl.-Ing. Rosemarie Helmerich, Oberregierungsrätin, BAM Fachgruppe VIII.2, Unter den Eichen 87, 12205 Berlin, Dr. Alain Nussbaumer, Stahlbaulehrstuhl (ICOM) der EPF Lausanne, GC B3 505, Station 18, CH-1015 Lausanne, Dr.-Ing. Hans-Peter Günther, Institut für Konstruktion und Entwurf der Universität Stuttgart, Paffenwaldring 7, 70569 Stuttgart, Dr.-Ing. Stefan Herion, Oberingenieur an der Versuchsanstalt für Stahl, Holz \& Steine der Universität Karlsruhe, Kaiserstrasse 12, 76128 Karlsruhe 